TRANSACTIONS OF THE

AMERICAN MATHEMATICAL SOCIETY

Volume 361, Number 12, December 2009, Pages 6583-6593

S 0002-9947(09)04762-X

Article electronically published on July 20, 2009

\title{
BROWN MEASURE \\ AND ITERATES OF THE ALUTHGE TRANSFORM FOR SOME OPERATORS ARISING FROM MEASURABLE ACTIONS
}

\author{
KEN DYKEMA AND HANNE SCHULTZ
}

\begin{abstract}
We consider the Aluthge transform $\widetilde{T}=|T|^{1 / 2} U|T|^{1 / 2}$ of a Hilbert space operator $T$, where $T=U|T|$ is the polar decomposition of $T$. We prove that the map $T \mapsto \widetilde{T}$ is continuous with respect to the norm topology and with respect to the $*-$ SOT topology on bounded sets. We consider the special case in a tracial von Neumann algebra when $U$ implements an automorphism of the von Neumann algebra generated by the positive part $|T|$ of $T$, and we prove that the iterated Aluthge transform converges to a normal operator whose Brown measure agrees with that of $T$ (and we compute this Brown measure). This proof relies on a theorem that is an analogue of von Neumann's mean ergodic theorem, but for sums weighted by binomial coefficients.
\end{abstract}

\section{INTRODUCTION}

For a Hilbert space $\mathcal{H}$, we let $\mathcal{B}(\mathcal{H})$ denote the set of bounded linear operators on $\mathcal{H}$. Let $T \in \mathcal{B}(\mathcal{H})$ and let $T=U|T|$ be its polar decomposition. The Aluthge transform of $T$ is the operator $\widetilde{T}=|T|^{1 / 2} U|T|^{1 / 2}$. This was first studied in [1 and has received much attention in recent years (see, for example, the papers cited below). One reason the Aluthge transform is interesting is in relation to the invariant subspace problem. Jung, Ko and Pearcy prove in [7] that $T$ has a nontrivial invariant subspace if and only if $\widetilde{T}$ does. They also note that when $T$ is a quasiaffinity, then $T$ has a nontrivial hyperinvariant subspace if and only if $\widetilde{T}$ does. (A quasiaffinity is an operator with zero kernel and dense range; the invariant and hyperinvariant subspace problems are interesting only for quasiaffinities.) Clearly, the spectrum of $\widetilde{T}$ equals that of $T$. Jung, Ko and Pearcy prove in [7] that other spectral data are also preserved by the Aluthge transform; see also 8 for more and related results.

The iterated Aluthge transforms (or Aluthge iterates) of $T$ are the operators $\widetilde{T}^{(k)}$, $k \geq 0$, defined by setting $\widetilde{T}^{(0)}=T$ and letting $\widetilde{T}^{(k+1)}$ be the Aluthge transform of $\widetilde{T}^{(k)}$. An interesting result, due to Yamazaki [14] (see also [13]), is that the spectral

Received by the editors December 12, 2005 and, in revised form, February 2, 2008

2000 Mathematics Subject Classification. Primary 47A05; Secondary 47 B99.

Key words and phrases. Aluthge transform, Brown measure, mean ergodic theorem.

An earlier version of this paper was distributed under the title: "On the Aluthge transform: continuity properties and Brown measure".

The first author's research was supported in part by NSF grant DMS-0300336.

As a student of the Ph.D. school OP-ALG-TOP-GEO, the second author was partially supported by the Danish Research Training Council and The Danish National Research Foundation.

(c)2009 American Mathematical Society Reverts to public domain 28 years from publication 
radius of $T$ is equal to the limit as $k \rightarrow \infty$ of the norm of $\widetilde{T}^{(k)}$. Recently, it was conjectured in [9] that $\widetilde{T}^{(k)}$ converges in the strong operator topology to a normal operator as $k \rightarrow \infty$. Antezana, Pujals and Stojanoff [2] proved this conjecture for operators on finite dimensional Hilbert spaces. While this conjecture has been shown in [4 not to hold in general, we believe, based partly on the evidence of examples described below, that it will hold for elements of finite von Neumann algebras.

The examples we consider are when $T=U|T|$, where $U$ is unitary and where the positive part $|T|$ belongs to an abelian algebra that is normalized by $U$ (i.e., on which $U$ acts by conjugation). In this situation, we describe the Aluthge iterates of $T$ and show that the conjecture mentioned above holds. We also calculate the Brown measure for these operators. Our proof relies on an analogue of von Neumann's mean ergodic theorem for sums weighted with binomial coefficients, and on a theorem of Haagerup and Schultz.

The authors thank the referee for identifying some needed improvements in the paper.

\section{Continuity properties in $\mathcal{B}(\mathcal{H})$}

The results of this section are applications of the continuous functional calculus for positive operators.

Lemma 2.1. For $n \in \mathbb{N}$ consider the function $f_{n}(t)=\sqrt{\max \left(\frac{1}{n}, t\right)}$ defined for real, nonnegative $t$ and let $A_{n}=f_{n}(|T|)$. Then

$$
\begin{aligned}
\left\|A_{n}\right\| & \leq \max \left(\frac{1}{\sqrt{n}},\|T\|^{1 / 2}\right), \\
\left\||T| A_{n}^{-1}\right\| & \leq\|T\|^{1 / 2}, \\
\left\|A_{n}-|T|^{1 / 2}\right\| & \leq \frac{1}{\sqrt{n}}, \\
\left\||T| A_{n}^{-1}-|T|^{1 / 2}\right\| & \leq \frac{1}{4 \sqrt{n}}, \\
\left\|A_{n} T A_{n}^{-1}-\widetilde{T}\right\| & \leq \frac{5}{4 \sqrt{n}}\|T\|^{1 / 2} .
\end{aligned}
$$

Proof. The norm bounds (1)-(4) follow easily from the continuous functional calculus. For (5), we use

$$
\begin{aligned}
\left\|A_{n} T A_{n}^{-1}-\widetilde{T}\right\| \leq & \left\|A_{n}-|T|^{1 / 2}\right\|\left\|U|T| A_{n}^{-1}\right\| \\
& +\left\||T|^{1 / 2} U\right\|\left\||T| A_{n}^{-1}-|T|^{1 / 2}\right\|
\end{aligned}
$$

and apply the previous estimates.

Lemma 2.2. Given $R \geq 1$ and $\epsilon>0$, there are real polynomials $p$ and $q$ such that for every $T \in \mathcal{B}(\mathcal{H})$ with $\|T\| \leq R$, we have

$$
\left\|\widetilde{T}-p\left(T^{*} T\right) T q\left(T^{*} T\right)\right\|<\epsilon
$$

Proof. Take $\epsilon<1$, for convenience. By Lemma 2.1. we may choose $n$ so large that

$$
\left\|\widetilde{T}-A_{n} T A_{n}^{-1}\right\|<\epsilon
$$

whenever $\|T\| \leq R$, where $A_{n}=f_{n}(|T|)$, with $f_{n}$ as in Lemma 2.1. Let $p$ and $q$ be real polynomials of one variable such that

$$
\max _{t \in[0, R]}\left|f_{n}(t)-p\left(t^{2}\right)\right|<\epsilon, \quad \max _{t \in[0, R]}\left|f_{n}(t)^{-1}-q\left(t^{2}\right)\right|<\epsilon .
$$


Then we have

$$
\left\|A_{n}-p\left(T^{*} T\right)\right\|<\epsilon, \quad\left\|A_{n}^{-1}-q\left(T^{*} T\right)\right\|<\epsilon .
$$

Using the estimates in Lemma 2.1, we get

$$
\begin{aligned}
& \left\|A_{n} T A_{n}^{-1}-p\left(T^{*} T\right) T q\left(T^{*} T\right)\right\| \\
& \quad \leq\left\|A_{n}-p\left(T^{*} T\right)\right\|\left\|T A_{n}^{-1}\right\|+\left\|p\left(T^{*} T\right) T\right\|\left\|A_{n}^{-1}-q\left(T^{*} T\right)\right\|<3 \epsilon R^{3 / 2} .
\end{aligned}
$$

Now replace $\epsilon$ in the above argument by $\epsilon /\left(3 R^{3 / 2}\right)$.

In the statements below, $\|\cdot\|$ refers to the operator norm topology and $*-$ SOT to the $*$-strong operator topology on $\mathcal{B}(\mathcal{H})$.

Theorem 2.3. The Aluthge transform map $T \mapsto \widetilde{T}$ is:

(a) $(\|\cdot\|,\|\cdot\|)$-continuous on $\mathcal{B}(\mathcal{H})$,

(b) (*-SOT,*-SOT)-continuous on bounded subsets of $\mathcal{B}(\mathcal{H})$.

Proof. For (a), let $T \in \mathcal{B}(\mathcal{H})$ and take $\epsilon \in(0,1]$. Let $R=\|T\|+1$ and let $p$ and $q$ be polynomials as found in Lemma 2.2 for these values of $R$ and $\epsilon$. Let $\delta \in(0,1]$ be such that $\|T-S\|<\delta$ implies

$$
\left\|p\left(T^{*} T\right) T q\left(T^{*} T\right)-p\left(S^{*} S\right) S q\left(S^{*} S\right)\right\|<\epsilon .
$$

Then $\|T-S\|<\delta$ implies

$$
\|\widetilde{T}-\widetilde{S}\|<\left\|\widetilde{T}-p\left(T^{*} T\right) T q\left(T^{*} T\right)\right\|+\left\|\widetilde{S}-p\left(S^{*} S\right) S q\left(S^{*} S\right)\right\|+\epsilon<3 \epsilon,
$$

where the last inequality is by choice of $p$ and $q$. This proves part (a).

For (b), let $R>1, \epsilon>0$ and let $p$ and $q$ be the polynomials found in Lemma 2.2 for these values. Let $x \in \mathcal{H}$. If $S, T \in \mathcal{B}(\mathcal{H})$, each with norm $\leq R$, then

$$
\begin{aligned}
\|(\widetilde{T}-\widetilde{S}) x\| & \leq 2 \epsilon\|x\|+\left\|\left(p\left(T^{*} T\right) T q\left(T^{*} T\right)-p\left(S^{*} S\right) S q\left(S^{*} S\right)\right) x\right\|, \\
\left\|(\widetilde{T}-\widetilde{S})^{*} x\right\| & \leq 2 \epsilon\|x\|+\left\|\left(p\left(T^{*} T\right) T^{*} q\left(T^{*} T\right)-p\left(S^{*} S\right) S^{*} q\left(S^{*} S\right)\right) x\right\| .
\end{aligned}
$$

Since multiplication is $*$-SOT continuous on bounded subsets of $\mathcal{B}(\mathcal{H})$, by taking $S$ in some neighborhood of $T$ in this topology, both quantities $\|\left(p\left(T^{*} T\right) T q\left(T^{*} T\right)-\right.$ $\left.p\left(S^{*} S\right) S q\left(S^{*} S\right)\right) x \|$ and $\left\|\left(p\left(T^{*} T\right) T^{*} q\left(T^{*} T\right)-p\left(S^{*} S\right) S^{*} q\left(S^{*} S\right)\right) x\right\|$ can be forced to be arbitrarily small. This proves (b).

\section{The Aluthge transform in finite von Neumann algebras}

In this section, we consider a von Neumann algebra $\mathcal{M}$ equipped with a normal, faithful, tracial state $\tau$, acting on the Hilbert space $\mathcal{H}:=L^{2}(\mathcal{M}, \tau)$, which is the completion of $\mathcal{M}$ with respect to the norm $\|x\|_{2}=\tau\left(x^{*} x\right)^{1 / 2}$. For $x \in \mathcal{M}$, we denote the corresponding element of $L^{2}(\mathcal{M}, \tau)$ by $\hat{x}$. Clearly, the Aluthge transform $\widetilde{T}$ of any $T \in \mathcal{M}$ also lies in $\mathcal{M}$. In $\mathcal{M}$, convergence in SOT in $B(\mathcal{H})$ implies convergence in $\|\cdot\|_{2}$, because $\|x\|_{2}=\|x \hat{1}\|_{\mathcal{H}}$. On the other hand, on bounded subsets of $\mathcal{M}$, convergence in $\|\cdot\|_{2}$ implies convergence in $*-\mathrm{SOT}$. Indeed, let $a_{n}$ be a sequence in the unit ball of $\mathcal{M}$ such that $\left\|a_{n}\right\|_{2} \rightarrow 0$ as $n \rightarrow \infty$, let $\eta \in L^{2}(\mathcal{M}, \tau)$, and let us show $\left\|a_{n} \eta\right\|_{\mathcal{H}} \rightarrow 0$ as $n \rightarrow \infty$. Let $\epsilon>0$. Then there is $x \in \mathcal{M}$ such that $\|\eta-\hat{x}\|_{\mathcal{H}}<\epsilon$. Letting $\rho$ denote the right action of $\mathcal{M}^{o p}$ on $\mathcal{H}$, we have

$$
\left\|a_{n} \eta\right\|_{\mathcal{H}} \leq 2 \epsilon+\left\|a_{n} \hat{x}\right\|_{\mathcal{H}}=2 \epsilon+\left\|\rho(x) a_{n} \hat{1}\right\| \mathcal{H} \leq 2 \epsilon+\|x\|\left\|a_{n}\right\|_{2}
$$


and from this we conclude $\left\|a_{n} \eta\right\|_{\mathcal{H}} \rightarrow 0$ as $n \rightarrow \infty$. Since $\left\|a_{n}^{*}\right\|_{2}=\left\|a_{2}\right\|_{2}$, we conclude $a_{n} \rightarrow 0$ in $*-$ SOT.

Therefore, we have the following immediate corollary of Theorem 2.3(b).

Theorem 3.1. The Aluthge transformation $T \mapsto \widetilde{T}$ is $\left(\|\cdot\|_{2},\|\cdot\|_{2}\right)$-continuous on bounded subsets of $\mathcal{M}$.

The proof of Theorem 2.2 of $[9$ ] carries over to the setting of finite von Neumann algebras to prove the following result.

Theorem 3.2. If $T \in \mathcal{M}$, then

$$
\|\widetilde{T}\|_{2} \leq\|T\|_{2}
$$

Moreover, equality holds if and only if $T$ is normal.

Proposition 3.3. Let $T \in \mathcal{M}$. If any subsequence $\left\{\widetilde{T}^{\left(n_{k}\right)}\right\}_{k=1}^{\infty}$ of the sequence of Aluthge interates of $T$ converges in $\|\cdot\|_{2}$ to a limit $L$, then $L$ is a normal operator.

Proof. By (6), $\left\|\widetilde{T}^{(n)}\right\|_{2}$ decreases to $\|L\|_{2}$ as $n \rightarrow \infty$. By Theorem 3.1. $\widetilde{T}^{\left(n_{k}+1\right)}$ converges in $\|\cdot\|_{2}$ as $k \rightarrow \infty$ to $\widetilde{L}$. Therefore, $\|\widetilde{L}\|_{2}=\|L\|_{2}$ and, by Theorem 3.2. $L$ is normal.

Lemma 3.4. Let $T \in \mathcal{M}$ and let $A_{n}=f_{n}(|T|)$ be as defined in Lemma 2.1. Let $P_{0} \in \mathcal{M}$ be the projection onto $\operatorname{ker} T$. Then

$$
\lim _{n \rightarrow \infty}\left\|A_{n}^{-1}|T|^{1 / 2}-\left(1-P_{0}\right)\right\|_{2}=0 .
$$

Proof. We have $A_{n}^{-1}|T|^{1 / 2}=h_{n}(|T|)$, where

$$
h_{n}(t)= \begin{cases}\sqrt{n} t^{1 / 2}, & t \in\left[0, \frac{1}{n}\right], \\ 1, & t \in\left[\frac{1}{n}, \infty\right) .\end{cases}
$$

Therefore, $0 \leq\left(1-P_{0}\right)-A_{n}^{-1}|T|^{1 / 2} \leq E_{|T|}\left(\left(0, \frac{1}{n}\right)\right)$, where $E_{|T|}$ is the spectral measure of $|T|$. Since $\left(E_{|T|}\left(\left(0, \frac{1}{n}\right)\right)\right)_{n=1}^{\infty}$ is a sequence of projections decreasing to zero as $n \rightarrow \infty$, we get (7).

The Brown measure [3] of an operator $T \in \mathcal{M}$ is defined as $\frac{1}{2 \pi}$ times the Laplacian of the function $\mathbb{C} \ni \lambda \mapsto \Delta(\lambda-T)$, where $\Delta$ is the Fuglede-Kadison determinant [6], defined by $\Delta(X)=\exp (\tau(\log |X|))$. By Theorem 4.3 of [3], the Brown measures $\mu_{T}$ and $\mu_{\widetilde{T}}$ are the same. In light of this, one would like in Proposition 3.3 to have that $L$ has the same Brown measure as $T$. This is at present unknown. However, we will conjecture even more than this, namely the actual convergence of Aluthge iterates.

Conjecture 3.5. Let $T \in \mathcal{M}$. Then the sequence $\left\{\widetilde{T}^{(n)}\right\}_{n=1}^{\infty}$ of Aluthge iterates of $T$ converges in $\|\cdot\|_{2}$ to a normal operator $N$ whose Brown measure is equal to the Brown measure of $T$.

This conjecture is the analogue for finite von Neumann algebras of Conjecture 5.6 of [9], which is about SOT-convergence of Aluthge iterates of $T \in \mathcal{B}(\mathcal{H})$. Although Cho, Jung and Lee [4] solve Conjecture 5.6 of [9] in the negative by giving an example of a weighted shift operator $T$ whose Aluthge iterates fail to converge even in the weak operator topology, that example clearly generates an infinite von Neumann algebra. As remarked earlier, this conjecture has recently been proved for matrices in [2]. 


\section{AN ERGODIC THEOREM FOR SUMS WEIGHTED WITH BINOMIAL COEFFICIENTS}

In this section, we prove a result that resembles von Neumann's mean ergodic theorem, but for sums that are weighted with binomial coefficients. Our proof is based on the proof of von Neumann's theorem found in 12. This theorem is covered in the case of a measure-preserving transformation by Theorem 1 of [11]. However, a check of the relevant conditions in our case seems to be just as much work as proving the theorem directly. After proving the theorem, we also draw some consequences that will be used in some examples in Section 5 .

Theorem 4.1. Let $T \in \mathcal{B}(\mathcal{H})$ satisfy $\|T\| \leq 1$. Then for every $v \in \mathcal{H}$, the vector

$$
H_{n}(v)=\frac{1}{2^{n}} \sum_{k=0}^{n}\left(\begin{array}{l}
n \\
k
\end{array}\right) T^{k} v
$$

converges as $n \rightarrow \infty$ to $P v$, where $P$ is the orthogonal projection of $\mathcal{H}$ onto its subspace $\operatorname{ker}(T-I)=\{x \in \mathcal{H} \mid T x=x\}$.

Proof. We have

$$
\operatorname{ker}(T-I)=\operatorname{ker}\left(T^{*}-I\right) .
$$

Indeed, it will suffice to show the inclusion $\subseteq$. If $T x=x$, then

$$
\begin{aligned}
0 \leq\left\|T^{*} x-x\right\|^{2} & =\left\|T^{*} x\right\|^{2}-\left\langle T^{*} x, x\right\rangle-\left\langle x, T^{*} x\right\rangle+\|x\|^{2} \\
& =\left\|T^{*} x\right\|^{2}-\langle x, T x\rangle-\langle T x, x\rangle+\|x\|^{2}=\left\|T^{*} x\right\|^{2}-\|x\|^{2} \leq 0,
\end{aligned}
$$

and (8) is proved. Therefore, $\operatorname{ker}(T-I)=\operatorname{ran}(T-I)^{\perp}$.

It is clear that if $v \in \operatorname{ker}(T-I)$, then $H_{n}(v)=v$ for all $n$. We will show that if $v \in \operatorname{ker}(T-I)^{\perp}$, then $\left\|H_{n}(v)\right\|$ converges to zero as $n \rightarrow \infty$. By linearity, this will imply that for general $v \in \mathcal{H}, H_{n}(v)$ converges to $P v$. In order to show that $\left\|H_{n}(v)\right\|$ converges to zero for $v \in \operatorname{ker}(T-I)^{\perp}$, it will suffice to show it for $v \in \operatorname{ran}(T-I)$, because this latter space is dense in $\operatorname{ker}(T-I)^{\perp}$ and each $H_{n}$ is a linear contraction. So we may assume $v=T y-y$, for some $y \in \mathcal{H}$. Then

$$
\begin{aligned}
H_{n}(v) & =\frac{1}{2^{n}} \sum_{k=0}^{n}\left(\begin{array}{l}
n \\
k
\end{array}\right)\left(T^{k+1} y-T^{k} y\right) \\
& =-\frac{1}{2^{n}} y+\frac{1}{2^{n}} \sum_{k=1}^{n}\left(\left(\begin{array}{c}
n \\
k-1
\end{array}\right)-\left(\begin{array}{l}
n \\
k
\end{array}\right)\right) T^{k} y+\frac{1}{2^{n}} T^{n+1} y .
\end{aligned}
$$

Since

$$
\left|\left(\begin{array}{c}
n \\
k-1
\end{array}\right)-\left(\begin{array}{l}
n \\
k
\end{array}\right)\right|=\frac{|2 k-n-1|}{k}\left(\begin{array}{c}
n \\
k-1
\end{array}\right),
$$

we get

$$
\left\|H_{n}(v)\right\| \leq \frac{1}{2^{n}}\left(2+\sum_{k=1}^{n} \frac{|2 k-n-1|}{k}\left(\begin{array}{c}
n \\
k-1
\end{array}\right)\right)\|y\| .
$$

It will suffice to show

$$
\lim _{n \rightarrow \infty} \frac{1}{2^{n}} \sum_{k=1}^{n} \frac{|2 k-n+1|}{k}\left(\begin{array}{l}
n \\
k
\end{array}\right)=0
$$


Let $0<\alpha<\frac{1}{2}$. Using Stirling's formula, it is easy to prove that for some constant $C>0$, we always have

$$
\sum_{k=n-\lfloor\alpha n-1\rfloor}^{n}\left(\begin{array}{l}
n \\
k
\end{array}\right)=\sum_{k=0}^{\lfloor\alpha n-1\rfloor}\left(\begin{array}{l}
n \\
k
\end{array}\right) \leq C n\left(\frac{1}{\alpha^{\alpha}(1-\alpha)^{(1-\alpha)}}\right)^{n}
$$

(and better estimates are possible; see, for example, [5]). Since

$$
\alpha^{\alpha}(1-\alpha)^{(1-\alpha)}>\frac{1}{2}
$$

we have

$$
\limsup _{n \rightarrow \infty} \frac{1}{2^{n}} \sum_{k=1}^{n} \frac{|2 k-n+1|}{k}\left(\begin{array}{l}
n \\
k
\end{array}\right)=\limsup _{n \rightarrow \infty} \frac{1}{2^{n}} \sum_{k=\lfloor\alpha n\rfloor}^{n-\lfloor\alpha n\rfloor} \frac{|2 k-n+1|}{k}\left(\begin{array}{l}
n \\
k
\end{array}\right) .
$$

However, for $\alpha n-1 \leq k \leq n-\alpha n+1$, we have

$$
\frac{|2 k-n+1|}{k} \leq \frac{(1-2 \alpha) n+3}{\alpha n-1}
$$

which together with (11) yields

$$
\limsup _{n \rightarrow \infty} \frac{1}{2^{n}} \sum_{k=1}^{n} \frac{|2 k-n+1|}{k}\left(\begin{array}{l}
n \\
k
\end{array}\right) \leq \frac{1-2 \alpha}{\alpha} .
$$

Taking $\alpha$ arbitrarily close to $\frac{1}{2}$ gives (9).

Let us now consider a probability space $(X, \mu)$ and a $\mu$-preserving, invertible transformation $\alpha: X \rightarrow X$. Let $E^{\alpha}: L^{1}(\mu) \rightarrow L^{1}(\mu)^{\alpha}$ denote the conditional expectation onto the subspace $L^{1}(\mu)^{\alpha}$ of $\alpha$-invariant functions in $L^{1}(\mu)$. In analogous notation, we will also write $E^{\alpha}: L^{2}(\mu) \rightarrow L^{2}(\mu)^{\alpha}$ and $E^{\alpha}: L^{\infty}(\mu) \rightarrow L^{\infty}(\mu)^{\alpha}$ for the restrictions of $E^{\alpha}$ to the indicated subspaces. For a random variable $b$ and $n \geq 0$, we will consider the random variable

$$
h_{n}(b)=\frac{1}{2^{n}} \sum_{k=0}^{n}\left(\begin{array}{l}
n \\
k
\end{array}\right) b \circ \alpha^{k}
$$

Applying Theorem 4.1 in the case $\mathcal{H}=L^{2}(\mu)$ and $T f=f \circ \alpha$, we have the following result.

Corollary 4.2. Let $b \in L^{2}(\mu)$ and let $c=E^{\alpha}(b)$. Then $\lim _{n \rightarrow \infty}\left\|h_{n}(b)-c\right\|_{2}=0$.

Corollary 4.3. Let $b \in L^{1}(X)$ and let $c=E^{\alpha}(b)$. Then $\lim _{n \rightarrow \infty}\left\|h_{n}(b)-c\right\|_{1}=0$.

Proof. For $M>0$, let $g_{M}:[-\infty, \infty] \rightarrow[-M, M]$ be

$$
g_{M}(t)= \begin{cases}-M, & t \leq-M \\ t, & -M \leq t \leq M \\ M, & M \leq t\end{cases}
$$

Let $\epsilon>0$. For $M>0$ sufficiently large, letting $\tilde{b}=g_{M} \circ b$ we have $\|\tilde{b}-b\|_{1}<\epsilon$, so $\left\|h_{n}(\tilde{b})-h_{n}(b)\right\|_{1}<\epsilon$. Moreover, we have $\|\tilde{c}-c\|_{1}<\epsilon$, where $\tilde{c}=E^{\alpha}(\tilde{b})$. Since 
we're working over a probability space, from Corollary 4.2 we have

$$
\lim _{n \rightarrow \infty}\left\|h_{n}(\tilde{b})-\tilde{c}\right\|_{1}=0
$$

and this yields

$$
\limsup _{n \rightarrow \infty}\left\|h_{n}(b)-c\right\|_{1} \leq 2 \epsilon
$$

We now consider a random variable $b: X \rightarrow[-\infty, R]$ for some real number $R$. Let $L^{\infty}(X)^{\alpha}$ denote the space of functions $f \in L^{\infty}(X)$ satisfying $f \circ \alpha=$ $f$. The conditional expectation of $b, E^{\alpha}(b): X \rightarrow[-\infty, R]$, is the unique $\alpha-$ invariant random variable such that $\int E^{\alpha}(b) f d \mu=\int b f d \mu \in[-\infty, R]$ whenever $f \in L^{\infty}(X)^{\alpha}$. Then the map $b \mapsto E^{\alpha}(b)$ is linear and order-preserving. Moreover, $b$ is integrable if and only if $E^{\alpha}(b)$ is integrable.

Corollary 4.4. Suppose $R$ is a real number and $b: X \rightarrow[-\infty, R]$ is measurable. Then $h_{n}(b)$ converges in probability to $E^{\alpha}(b)$ as $n \rightarrow \infty$, where we take the obvious metric on $[-\infty, R]$.

Proof. Fix $K>0$ large and $\epsilon>0$ and $\delta>0$ small. Consider the $\alpha$-invariant set $F=\left\{\omega \mid E^{\alpha}(b)(\omega) \geq-K\right\}$. Then $E^{\alpha}\left(1_{F} b\right)=1_{F} E^{\alpha}(b)$ is integrable, so $1_{F} b$ is integrable. By Corollary 4.3, $\lim _{n \rightarrow \infty}\left\|h_{n}\left(1_{F} b\right)-E^{\alpha}\left(1_{F} b\right)\right\|_{1}=0$. Also, we have $h_{n}\left(1_{F} b\right)=1_{F} h_{n}(b)$. Thus, for $n$ sufficiently large we have

$$
\mu\left(\left\{\omega\left|E^{\alpha}(b)(\omega) \geq-K,\right| h_{n}(b)(\omega)-E^{\alpha}(b)(\omega) \mid \geq \epsilon\right\}\right)<\delta .
$$

For integers $m$ with $m>|R|$ and with $g_{m}$ as defined in (13), we have $E^{\alpha}\left(g_{m} \circ b\right) \geq$ $E^{\alpha}\left(g_{m+1} \circ b\right) \geq E^{\alpha}(b)$ and we claim that $E^{\alpha}\left(g_{m} \circ b\right)$ converges a.e. as $m \rightarrow \infty$ to $E^{\alpha}(b)$. Indeed, let $H=\lim _{m \rightarrow \infty} E^{\alpha}\left(g_{m} \circ b\right)$. Choosing $f \in L^{\infty}(X)^{\alpha}$ nonnegative, we have by monotone convergence,

$$
\begin{aligned}
\int E^{\alpha}(b) f d \mu=\int b f d \mu & =\lim _{m \rightarrow \infty} \int\left(g_{m} \circ b\right) f d \mu \\
& =\lim _{m \rightarrow \infty} \int E^{\alpha}\left(g_{m} \circ b\right) f d \mu=\int H f d \mu .
\end{aligned}
$$

This implies $H=E^{\alpha}(b)$ a.e.

By Egoroff's Theorem, $\exp \left(E^{\alpha}\left(g_{m} \circ b\right)\right)$ converges in measure to $\exp \left(E^{\alpha}(b)\right)$, so $E^{\alpha}\left(g_{m} \circ b\right)$ converges in measure to $E^{\alpha}(b)$. Therefore, for some $m$ we have

$$
\mu\left(\left\{\omega \mid E^{\alpha}(b)(\omega)<-K, E^{\alpha}\left(g_{m} \circ b\right)(\omega) \geq-K+1\right\}\right)<\delta .
$$

Again by Corollary 4.3. $\lim _{n \rightarrow \infty}\left\|h_{n}\left(g_{m} \circ b\right)-E^{\alpha}\left(g_{m} \circ b\right)\right\|_{1}=0$, so for $n$ sufficiently large we have

$$
\mu\left(\left\{\omega \mid E^{\alpha}(b)(\omega)<-K, h_{n}\left(g_{m} \circ b\right) \geq-K+2\right\}\right)<2 \delta .
$$

Finally, since $h_{n}(b) \leq h_{n}\left(g_{m} \circ b\right)$, for $n$ sufficiently large we have

$$
\mu\left(\left\{\omega \mid E^{\alpha}(b)(\omega)<-K, h_{n}(b) \geq-K+2\right\}\right)<2 \delta .
$$

Combining (14) and (15) finishes the proof.

Corollaries 4.3 and 4.4 are, of course, straightforward consequences of Theorem 4.1. Analogues where von Neumann's mean ergodic theorem is used instead 
of Theorem 4.1 are obtained similarly. For future use, we state the following such analogue of Corollary 4.4.

Proposition 4.5. Suppose $R$ is a real number and $b: X \rightarrow[-\infty, R]$ is measurable. Then the random variable

$$
\frac{1}{n} \sum_{k=0}^{n-1} b \circ \alpha^{k}
$$

converges in probability to $E^{\alpha}(b)$ as $n \rightarrow \infty$.

\section{Some examples in finite von Neumann algebras}

Let $T \in \mathcal{B}(\mathcal{H})$ and let $T=U|T|$ be its polar decomposition. The Aluthge transform of $T$ is by definition $\widetilde{T}=|T|^{1 / 2} U|T|^{1 / 2}$. It is easily seen that if $V \in \mathcal{B}(\mathcal{H})$ is any partial isometry whose restriction to $U^{*} U \mathcal{H}$ equals $U$, then $\widetilde{T}=|T|^{1 / 2} V|T|^{1 / 2}$. In particular, if $T$ belongs to a finite von Neumann algebra, then we may take $V$ to be a unitary extension of $U$.

We consider a probability space $(X, \mu)$ and we let $\alpha$ be an invertible, measurepreserving transformation of $X$. Let $\mathcal{M}=L^{\infty}(X) \rtimes_{\alpha} \mathbb{Z}$ be the crossed product von Neumann algebra, which is, therefore, generated by a copy of $L^{\infty}(X)=L^{\infty}(X, \mu)$ and a unitary $U$ such that for $f \in L^{\infty}(X)$, we have $U f U^{*}=f \circ \alpha$. For convenience, we will write

$$
\tilde{\alpha}(f)=f \circ \alpha^{-1}=U^{*} f U,
$$

so that we have $f U=U \tilde{\alpha}(f)$. The set

$$
\operatorname{span}\left\{U^{k} f \mid k \in \mathbb{Z}, f \in L^{\infty}(X)\right\}
$$

is then strongly dense in $\mathcal{M}$, and there is a normal tracial state $\tau$ on $\mathcal{M}$ uniquely determined by

$$
\tau\left(U^{k} f\right)=\delta_{k, 0} \int_{X} f \mathrm{~d} \mu, \quad k \in \mathbb{Z}, f \in L^{\infty}(X) .
$$

We will investigate the Aluthge iterates of operators of the form $T=U|T| \in \mathcal{M}$ with $U$ as above and with $|T| \in L^{\infty}(X)$.

Lemma 5.1. The $n$-th Aluthge iterate of $T$ is

$$
\widetilde{T}^{(n)}=U\left|\widetilde{T}^{(n)}\right|,
$$

where

$$
\left|\widetilde{T}^{(n)}\right|=\prod_{k=0}^{n} \tilde{\alpha}^{k}\left(|T|^{\left(\begin{array}{c}
n \\
k
\end{array}\right) / 2^{n}}\right) .
$$

Proof. The proof proceeds by induction over $n$. Clearly, (17) and (18) hold for $n=0$ (with the convention that $\widetilde{T}^{(0)}=T$ ). Let $N \geq 1$, and assume that (18) holds 
for $n=N-1$. Then for the $N$ th Aluthge iterate we have:

$$
\begin{aligned}
\widetilde{T}^{(N)} & =\left[\prod_{k=0}^{N-1} \tilde{\alpha}^{k}\left(|T|^{\left.\left(\begin{array}{c}
N-1 \\
k
\end{array}\right) / 2^{N-1}\right)}\right]^{\frac{1}{2}} U\left[\prod_{l=0}^{N-1} \tilde{\alpha}^{l}\left(|T|^{\left.\left(\begin{array}{c}
N-1 \\
l
\end{array}\right) / 2^{N-1}\right)}\right)\right]^{\frac{1}{2}}\right. \\
& =\left[\prod_{k=0}^{N-1} \tilde{\alpha}^{k}\left(|T|^{\left.\left(\begin{array}{c}
N-1 \\
k
\end{array}\right) / 2^{N}\right)}\right)\right]\left[\prod_{l=0}^{N-1} \tilde{\alpha}^{l}\left(|T|^{\left.\left(\begin{array}{c}
N-1 \\
l
\end{array}\right) / 2^{N}\right)}\right]\right. \\
& =U\left[\prod_{k=0}^{N-1} \tilde{\alpha}^{k+1}\left(|T|^{\left(\begin{array}{c}
N-1 \\
k
\end{array}\right) / 2^{N}}\right)\right]\left[\prod_{l=0}^{N-1} \tilde{\alpha}^{l}\left(|T|^{\left.\left(\begin{array}{c}
N-1 \\
l
\end{array}\right) / 2^{N}\right)}\right]\right. \\
& =U\left[\prod_{k=1}^{N} \tilde{\alpha}^{k}\left(|T|^{\left.\left(\begin{array}{c}
N-1 \\
k-1
\end{array}\right) / 2^{N}\right)}\right)\right]\left[\prod_{l=0}^{N-1} \tilde{\alpha}^{l}\left(|T|^{\left.\left(\begin{array}{c}
N-1 \\
l
\end{array}\right) / 2^{N}\right)}\right)\right] \\
& =U \tilde{\alpha}^{N}\left(|T|^{\left.\left(\begin{array}{c}
N-1 \\
N-1
\end{array}\right) / 2^{N}\right)}\left[\prod_{k=1}^{N-1} \tilde{\alpha}^{k}\left(|T|^{\left.\left(\begin{array}{c}
N-1 \\
k-1
\end{array}\right) / 2^{N}+\left(\begin{array}{c}
N-1 \\
k
\end{array}\right) / 2^{N}\right)}\right)\right]|T|^{\frac{1}{2^{N}}}\right. \\
& =U \prod_{k=0}^{N} \tilde{\alpha}^{k}\left(|T|^{\left.\left(\begin{array}{c}
N \\
k
\end{array}\right) / 2^{N}\right)},\right.
\end{aligned}
$$

and this shows that (17) and (18) hold for $n=N$ as well.

Given a random variable $b: X \rightarrow[-\infty, R]$ for some real number $R$, we let $E^{\alpha}(b)$ denote the conditional expectation of $b$ onto the space of such $\alpha$-invariant random variables, as described immediately before Corollary 4.4 .

Theorem 5.2. Let $T=U|T|$ be as described above. Then

$$
\lim _{n \rightarrow \infty}\left\|\widetilde{T}^{(n)}-U H\right\|_{2}=0,
$$

where $H=\exp \left(E^{\alpha}(\log (|T|))\right)$.

Proof. In light of (17), it will suffice to show $\left|\widetilde{T}^{(n)}\right| \stackrel{\|\cdot\|_{2}}{\longrightarrow} H$ as $n \rightarrow \infty$. Since the Aluthge iterates $\left(\widetilde{T}^{(n)}\right)_{n=1}^{\infty}$ form a norm-bounded sequence in $L^{\infty}(X)$, it clearly suffices to show that $\left|\widetilde{T}^{(n)}\right|$ converges in probability to $H$. This is equivalent to convergence in probability of $\log \left|\widetilde{T}^{(n)}\right|$ to $E^{\alpha}(\log (|T|))$. From (18),

$$
\log \left|\widetilde{T}^{(n)}\right|=\frac{1}{2^{n}} \sum_{k=0}^{n}\left(\begin{array}{l}
n \\
k
\end{array}\right) \log (|T|) \circ \alpha^{k}=h_{n}(\log (|T|)),
$$

with $h_{n}$ as defined in (12). Now the required convergence follows from Corollary 4.4.

If $\alpha$ is ergodic, then $E^{\alpha}(b)=\tau(b)$ and we have the following.

Corollary 5.3. If $T=U|T|$ as above and if $\alpha$ is ergodic, then the Aluthge iterates $\left(\widetilde{T}^{(n)}\right)_{n=1}^{\infty}$ converge in $\|\cdot\|_{2}$ to $\Delta(T) U$, where $\Delta(T) \in[0, \infty)$ is the Fuglede-Kadison determinant of $T$.

We will now verify Conjecture 3.5 for our operators $T$ by proving that the Brown measure of $T$ is the same as that of $U H$, with $H$ as in Theorem 5.2 This is an application of very general results from [10]. 
Theorem 5.4. Let $T=U|T|$ and $H=\exp \left(E^{\alpha}(\log |T|)\right)$ be as in Theorem [5.2, Then in the strong operator topology,

$$
\left[\left(T^{m}\right)^{*} T^{m}\right]^{\frac{1}{2 m}} \stackrel{m \rightarrow \infty}{\longrightarrow} H
$$

and

$$
\left[T^{m}\left(T^{m}\right)^{*}\right]^{\frac{1}{2 m}} \stackrel{m \rightarrow \infty}{\longrightarrow} H .
$$

Moreover, $U H$ is a normal operator and the Brown measures of $T$ and $U H$ agree.

Proof. We will prove (19). Note that

$$
T^{m}=U^{m} \prod_{k=0}^{m-1} \tilde{\alpha}^{k}(|T|),
$$

so that

$$
\left[\left(T^{m}\right)^{*} T^{m}\right]^{\frac{1}{2 m}}=\left[\prod_{k=0}^{m-1} \tilde{\alpha}^{k}(|T|)\right]^{\frac{1}{m}}
$$

and

$$
\log \left[\left(T^{m}\right)^{*} T^{m}\right]^{\frac{1}{2 m}}=\frac{1}{m} \sum_{k=0}^{m-1} \log \left(\tilde{\alpha}^{k}(|T|)\right) .
$$

By Proposition 4.5, the random variable $\log \left[\left(T^{m}\right)^{*} T^{m}\right]^{\frac{1}{2 m}}$ converges in probability to $E^{\alpha}(\log |T|)$, which implies convergence in probability of $\left[\left(T^{m}\right)^{*} T^{m}\right]^{\frac{1}{2 m}}$ to $H$. Since the sequence of random variables $\left(\left[\left(T^{m}\right)^{*} T^{m}\right]^{\frac{1}{2 m}}\right)_{m \in \mathbb{N}}$ is uniformly bounded, convergence in probability implies converges in $\|\cdot\|_{2}$ and, hence, also in SOT to $H$. The SOT-convergence (20) follows analogously.

Since $H$ is $\alpha$-invariant, $H$ commutes with $U$ and $U H$ is normal. We will now show that the Brown measures $\mu_{T}$ and $\mu_{U H}$ agree. First note that both measures are invariant under rotations. Indeed, for $\theta \in \mathbb{R}$ we have an isomorphism $\pi_{\theta}: \mathcal{M} \rightarrow \mathcal{M}$ given by

$$
\begin{aligned}
\pi_{\theta}(U) & =e^{i \theta} U, \\
\pi_{\theta}(f) & =f, \quad f \in L^{\infty}(X) .
\end{aligned}
$$

It is also clear from (16) that $\pi_{\theta}$ preserves the trace so that

$$
\mu_{T}=\mu_{\pi(T)}=\mu_{e^{i \theta} T} \quad \text { and } \quad \mu_{U H}=\mu_{\pi(U H)}=\mu_{e^{i \theta} U H} .
$$

Hence, it suffices to show that the two measures agree on the closed disks centered at the origin, $\overline{B(0, r)}$, for $r>0$. Since $U H$ is normal,

$$
\mu_{U H}(\overline{B(0, r)})=\tau\left(1_{[0, r]}(H)\right) .
$$

On the other hand, applying Thm. 8.1 of [10], the convergence (19) and Lemma 7.16(iii) of 10, we get

$$
\mu_{T}(\overline{B(0, r)})=\tau\left(1_{[0, r]}(H)\right) .
$$




\section{REFERENCES}

[1] A. Aluthge, 'On $p$-hyponormal operators for $0<p<1$,' Integr. Equ. Oper. Theory 13 (1990), 307-315. MR1047771 (91a:47025)

[2] J. Antezana, E.R. Pujals and D. Stojanoff, 'The iterated Aluthge transforms of a matrix converge,' preprint, arXiv:0711.3727.

[3] L.G. Brown, 'Lidskii's Theorem in the type II case,' Geometric methods in operator algebras (Kyoto, 1983), H. Araki and E.G. Effros (eds.), Pitman Res. Notes Math. Ser. 123, Longman Sci. Tech., 1986, pp. 1-35. MR866489 (88d:47024)

[4] M. Cho, I. Jung and W. Lee, 'On Aluthge transforms of $p$-hyponormal operators,' Integr. Equ. Oper. Theory, 53 (2005), 321-329. MR2186093(2006m:47034)

[5] J. Elton, 'Sign-embeddings of $\ell_{1}^{n}$,' Trans. Amer. Math. Soc. 279 (1983), 113-124. MR704605 (84g:46023)

[6] B. Fuglede and R.V. Kadison, 'Determinant theory in finite factors,' Ann. of Math. (2) 55 (1952), 520-530. MR0052696 (14:660a)

[7] I. Jung, E. Ko and C. Pearcy, 'Aluthge transforms of operators,' Integr. Equ. Oper. Theory 37 (2000), 437-448. MR1780122 (2001i:47035)

[8] I. Jung, E. Ko and C. Pearcy, 'Spectral pictures of Aluthge transforms of operators,' Integr. Equ. Oper. Theory 40 (2001), 52-60. MR1829514 (2002b:47007)

[9] I. Jung, E. Ko and C. Pearcy, 'The iterated Aluthge transform of an operator,' Integr. Equ. Oper. Theory 45 (2003), 375-387. MR.1971744 (2004b:47036)

[10] U. Haagerup and H. Schultz, 'Invariant subspaces for operators in a general $\mathrm{II}_{1}$-factor', preprint, arXiv:math.OA/0611256.

[11] D.L. Hanson and G. Pledger, 'On the mean ergodic theorem for weighted averages', $Z$. Wahrscheinlichkeitstheorie verw. Geb. 13 (1969), 141-149. MR0254214 (40:7423)

[12] K. Petersen, Ergodic Theory, Cambridge University Press, Cambridge, 1983. MR833286 (87i:28002)

[13] D. Wang, 'Heinz and McIntosh inequalities, Aluthge transformation and the spectral radius,' Math. Inequal. Appl. 6 (2003), 121-124. MR1950656(2003k:47008)

[14] T. Yamazaki, 'An expression of spectral radius via Aluthge transformation,' Proc. Amer. Math. Soc. 130 (2002), 1131-1137. MR 1873788(2002i:47005)

Department of Mathematics, Texas A\&M University, College Station, Texas 778433368

E-mail address: kdykema@math.tamu.edu

Department of Mathematics and Computer Science, University of Southern Denmark, Campusvej 55, 5230 Odense M, Denmark

E-mail address: schultz@imada.sdu.dk 